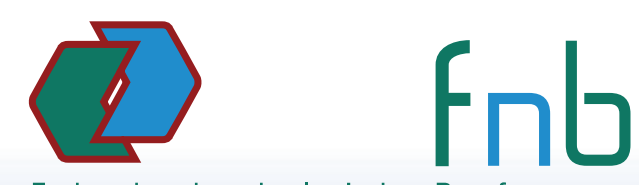

Fachverband nephrologischer Berufsgruppen

www. nephro-fachverband.de

\title{
10. Dreiländer Kongress: 17.-19.11.2016 in Konstanz Motto „Wissenschaft = Wissen schaffen = Wissen anwenden“
}

2016 findet der 10. Dreiländer Kongress in Konstanz statt. Vom 17.-19.11. halten nephrologische Pflegekräfte Vorträge für Pflegende unter dem Motto „Wissenschaft = Wissen schaffen = Wissen anwenden“. Der fnb ruft dazu auf, Abstracts und Poster einzureichen - der Kongress lebt von der Mitgestaltung durch Pflegekräfte.

Das Jubiläum des 10. Dreiländer Kongresses (DLK) 2016 war für uns Anlass, bei einem der Gründungsväter, Alois Gorke, nachzufragen, wie der DLK entstanden ist. Er berichtete, dass auf einem Strategieseminar der EDTNA/ERCA im Jahr 1993, an welchem neben Alois Gorke auch Waltraud Küntzle und Franz Techert teilgenommen haben, unter anderem die Idee eines europäischen Regionalkongresses in einer Sprache geboren wurde. Die konsequente Umsetzung dieser Idee war der 1. Dreiländer Kongress 1998 in Konstanz unter der Leitung von Christa Tast, Deutschland, Doris Rosenkranz, Österreich, und Ursula Elfrich, Schweiz.

\section{Machen Sie mit}

Die Idee, einen Kongress zu veranstalten, an dem Pflegende Vorträge für ihre Kollegen halten, ist bis heute die Grundlage für den DLK und macht diesen dadurch zu
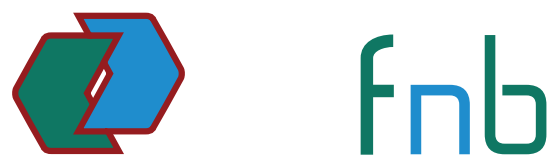

Fachverband nephrologischer Berufsgruppen

Geschäftsstelle fnb, Uschi Gaspar

In den Beunen 6, 65479 Raunheim,

Tel.: 06142/408549, Fax: 06142/408551

E-Mail: uschi.gaspar@nephro-fachverband.de,

www.nephro-fachverband.de

Vorstand des fnb:

- Michael Reichardt (1. Vorsitzender)

- Jürgen Berner (2. Vorsitzender)

- Ina Wiegard-Szramek (Schatzmeisterin)

- Marvin Breckling (Schriftführer) einem wichtigen Forum für die nephrologische Pflege. Deshalb hier unser Aufruf an Sie: Lassen Sie Kollegen aus dem deutschsprachigen Raum der 3 Länder Schweiz, Österreich und Deutschland an Ihren Erfahrungen und Ihrem Fachwissen teilhaben. Stellen Sie Ihre Haus-, Fachund Projektarbeiten sowie Studien vor oder berichten Sie über den „besonderen Fall“ aus Ihrer täglichen Praxis.

\section{Themenschwerpunkte}

Die Themenschwerpunkte sind:

- Sicherheit in der nephrologischen Pflege (alles rund um Qualität, Technik, Notfälle, Ausbildung)

- Dialysezugänge (in der HD und PD)

- Prävention/Gesundheitsförderung (sowohl für Patienten als auch für Personal; alles rund um die Beratung und den Sport an der Dialyse)

- Berufspolitik (alles zur Wirtschaftlichkeit, Finanzierung von Nierenersatzverfahren, Entwicklung von Berufsgruppen)

- Wissenschaft in der nephrologischen Pflege (wie z.B.: Wieviel Wissenschaft ist noch tragbar?, Wissen vermitteln, Wissen implementieren, Wissen schaffen)

- Freies Forum (keine festgelegten Themen: Berichten Sie über etwas, das Sie für interessant halten)

Die Themenschwerpunkte sind als grobe Struktur zu verstehen. Ihre Neugierde, Ihr wissenschaftliches Arbeiten und Ihre
Fantasie bei der Gestaltung Ihrer Arbeit sollen die Grundlage zur Interpretation der Themen sein.

\section{Posterausstellung und Abstracts} 2016 wollen wir zum ersten Mal eine Posterausstellung auf dem DLK ausrichten. Sie haben deshalb auch die Möglichkeit, Ihre Arbeit auf einem Poster zu präsentieren. Reichen Sie hierfür ein Abstract Ihres Posters bei uns ein. Ideal wäre es, wenn Sie Ihr Poster im Rahmen einer Posterbegehung auf dem DLK persönlich vorstellen. Das am besten bewertete Abstract wird mit einem Geldpreis vom 500 Euro prämiert, das am besten bewertete Poster wird mit 200 Euro belohnt.

\section{Weitere Informationen}

Auf der Homepage (www.nephro-fachver band.de/dreilaender-kongress-konstanz. $\mathrm{html}$ ) finden Sie eine Abstractvorlage und eine Anleitung („Wie schreibe ich ein Abstract?" und „Wie gestalte ich ein Poster?“). Ihr Poster können Sie online oder per Post bis zum 01.02.2016 einreichen. Abstracts senden Sie bitte an die auf der Homepage angegebene Adresse. Für weitere Hilfestellungen oder die Beantwortung von Fragen stehen wir Ihnen unter den im Kasten angegebenen Kontaktdaten gerne zur Verfügung. Wir freuen uns, von Ihnen zu hören und zu lesen, sowie auf Ihr Abstract.

Ina Wiegard-Szramek, Essen;

Martha Girak, Wien (Österreich)

\section{Kontaktdaten}

Martha Girak

(Kongresspräsidentin)

martha.girak@akhwien.at

Ina Wiegard-Szramek (Programmverantwortliche) ina.wiegard@nephro-fachverband.de 